\title{
Aneurysmal Bone Cyst Arising from the First Rib: A Rare Cause of Thoracic Outlet Syndrome
}

\author{
Manuel Medina ${ }^{1}$ Subroto Paul ${ }^{2}$ \\ ${ }^{1}$ Department of Surgery, New York Presbyterian/Queens, Flushing, \\ New York, United States \\ 2 Department of Cardiothoracic Surgery, New York Presbyterian \\ Hospital, New York, New York, United States \\ Thorac Cardiovasc Surg Rep 2016;5:74-76.
}

\begin{abstract}
Address for correspondence Manuel Medina, MD, New York Presbyterian/Queens, 5645 Main St. Flushing, New York 11357, United States (e-mail: mam9400@nyp.org).
\end{abstract}

\begin{abstract}
Aneurysmal bone cyst is a rare benign cystic bone lesion with an incidence of only 0.14 per 100,000 individuals and most commonly affects the metaphyses of long bones, spine, and pelvis. We present a very rare case of a 17 -year-old boy with a rapidly

Keywords

- thoracic outlet

- chest wall

- aneurysmal bone cyst expanding aneurysmal bone cyst arising from the first rib, resulting in neurogenic thoracic outlet syndrome secondary to its compression of the brachial plexus. The patient's symptoms resolved after en bloc resection. To our knowledge there have been no other reports in the literature of thoracic outlet syndrome due to aneurysmal bone cyst arising from the first rib.
\end{abstract}

\section{Introduction}

Thoracic outlet syndrome is caused by compression of neurovascular structures within the subclavicular space. Compression can occur in the interscalene, costoclavicular, or subpectoral minor spaces secondary to trauma or congenital anomalies. Congenital anomalies are less frequent and consist of fibromuscular bands, variant anatomy of scalene muscles, cervical ribs, and, even rarer, thoracic ribs. ${ }^{1}$ However, to our knowledge there have been no reports in the literature of thoracic outlet syndrome due to aneurysmal bone cyst (ABC) arising from the first rib.

\section{Case Description}

A 17-year-old boy was evaluated for left-sided neck pain as well as left hand numbness and weakness experienced over a 3-month period. On examination, a firm, fixed mass was palpated in the left neck. A chest computed tomography (CT) and subsequent magnetic resonance imaging (MRI) demonstrated an $11.7 \times 7.3 \times 6.5 \mathrm{~cm}$ complex mass. T2-weighted coronal MRI images ( - Fig. 1A) demonstrated multiple fluidfilled levels within the expansile bone lesion emanating from the left first rib. Sagittal images ( - Fig. 1B) demonstrated anterior displacement of the brachial plexus and subclavian vessels, as well as compression of the jugular vein. Axial images ( - Fig. 1C) demonstrated subclavian vein compression. Although a CT-guided biopsy was inconclusive, the mass was thought to be consistent with an $\mathrm{ABC}$. The mass was resected through a left hemi-clamshell incision necessitating clavicular resection and reimplantation for additional exposure of the subclavian vessels.

An incision was made overlying the clavicle from the sternoclavicular to the tracheloclavicular joint. The soft tissues were dissected and the clavicle was subsequently resected. The mass was evident, and found to be displacing the brachial plexus as well as the left subclavian artery and vein anteriorly. The subclavian artery and vein were tediously dissected off the mass. Then, the first rib was resected and displaced posteriorly. However, dissection was further limited by the large size of the mass and inability to define the exact anatomical plane of the brachial plexus. For greater exposure, a left clamshell incision was performed, and the mass was readily apparent at this point.

There were partial bone fragments in the mass, consistent with an ABC. Subsequently, the dissection was carried anteriorly while displacing the mass posteriorly into the chest, such that the brachia plexus was easily defined and dissected received

September 8, 2015

accepted

October 19, 2015

published online

December 16, 2015
DOI http://dx.doi.org/

10.1055/s-0035-1569994. ISSN 2194-7635. (c) 2016 Georg Thieme Verlag KG
Stuttgart · New York

License terms

()(1) $\Theta \circledast$ 


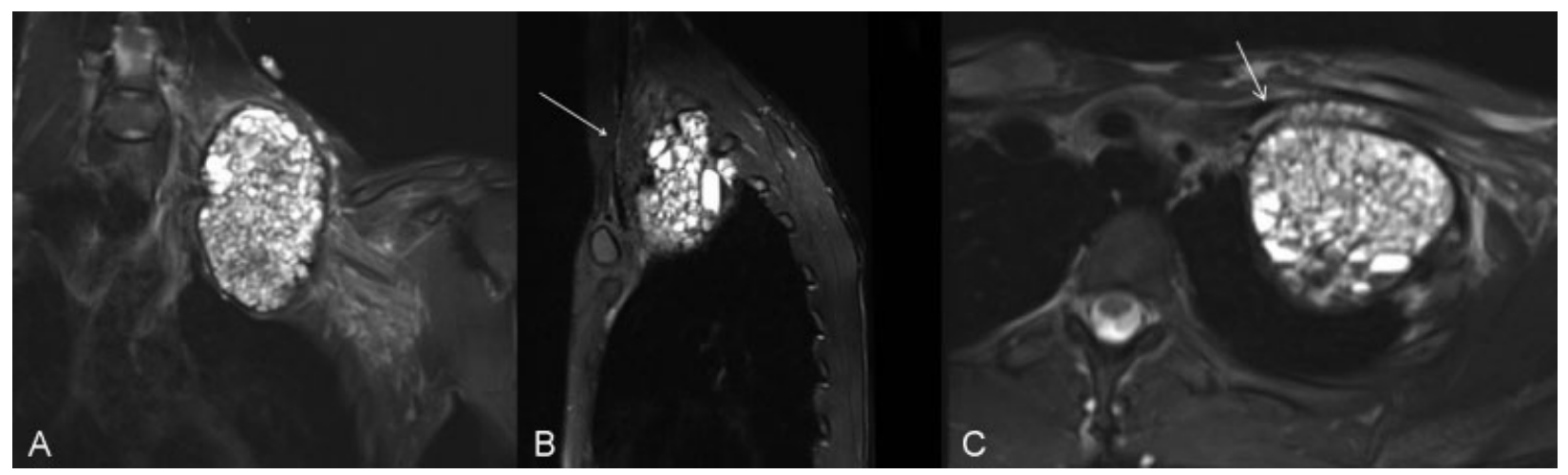

Fig. 1 T2-weighted MRI showing multiple fluid-fluid levels within the expansile bone lesion emanating from the left first rib with extension into neck (A) and compression of jugular (B) and subclavian vessels (C).

off the mass. Neural monitoring did not reveal any evidence of any injury to any of the nerves. Subsequently, the mass was resected in its entirety up to its apical portion that was extending into the left neck, close to the origin of the brachial plexus. At this point, the mass was partially excised, with over $99 \%$ of the mass being removed and sent for permanent section, as it appeared to be an ABC ( - Fig. 2A). The remaining fragments of the bone-like particles which were adherent to the brachial plexus were removed in pieces, such that the brachial plexus was not injured. The patient's left neck pain and hand weakness resolved after resection. The final pathology was consistent with an $\mathrm{ABC}$ (-Fig. 2B, 2C).

\section{Discussion}

$A B C s$ are very rare benign bone tumors with a reported annual incidence of 0.14 per 100,000 individuals, ${ }^{2}$ occurring most commonly in the metaphyses of long bones (femur $22 \%$, tibia $17 \%$, humerus $10 \%$, fibula $9 \%$ ), posterior elements of vertebral bodies (15\%), and pelvis (9\%). ${ }^{3} \mathrm{ABC}$ is an expanding osteolytic bone lesion consisting of blood-filled spaces, separated by connective tissue septae containing trabeculae of bone or osteoid tissue and osteoclast giant cells. A condition of the first three decades, approximately three-quarters of the cases observed affect persons younger than 20 years of age and are rarely seen in persons after the age of 30 . When arising from ribs, $A B C s$ usually involve the posterior or lateral aspects and have been shown to affect any rib except the lower three. They are associated with pain (46\%), palpable mass (21\%), dyspnea (7\%), or pathologic fracture (7\%). ${ }^{4}$ Our patient presented in atypical fashion from what has been reported. While he complained of pain and had a large palpable mass, both were located in his left neck, not in the posterolateral regions of thorax as described in the literature. $\mathrm{He}$ also complained of left hand numbness and weakness, constellation of symptoms comprising thoracic outlet syndrome and thus suggesting involvement of the brachial plexus. The subsequent MRI performed to further characterize the mass showed its typical fluid-filled levels within a cyst arising anteriorly from the first rib and its internal septations, with soap bubble features.

Multiple treatment modalities exist for ABCs including curettage, resection, intracystic injections, and embolization. Cottalorda and Bourelle ${ }^{5}$ performed a review of literature of over 1,200 ABCs and analyzed various treatment modalities. In summary, there is no set algorithm for treating these lesions and thus treatment must be tailored to each individual. Location, size, neurovascular involvement, and age of patient must be taken into consideration when choosing therapy to decrease morbidity and chance of recurrence. The authors, however, state that the overall recurrence rate is lowest with en bloc resection of the tumor. Given the rapid evolution of our patient's symptoms and the risk of developing venous thrombosis in jugular and subclavian veins from extrinsic compression or further compromise of the brachial plexus, we did not attempt to decrease cyst size or vascularity via intracystic injection or arterial embolization. Both of these interventions would require several weeks for full effect to

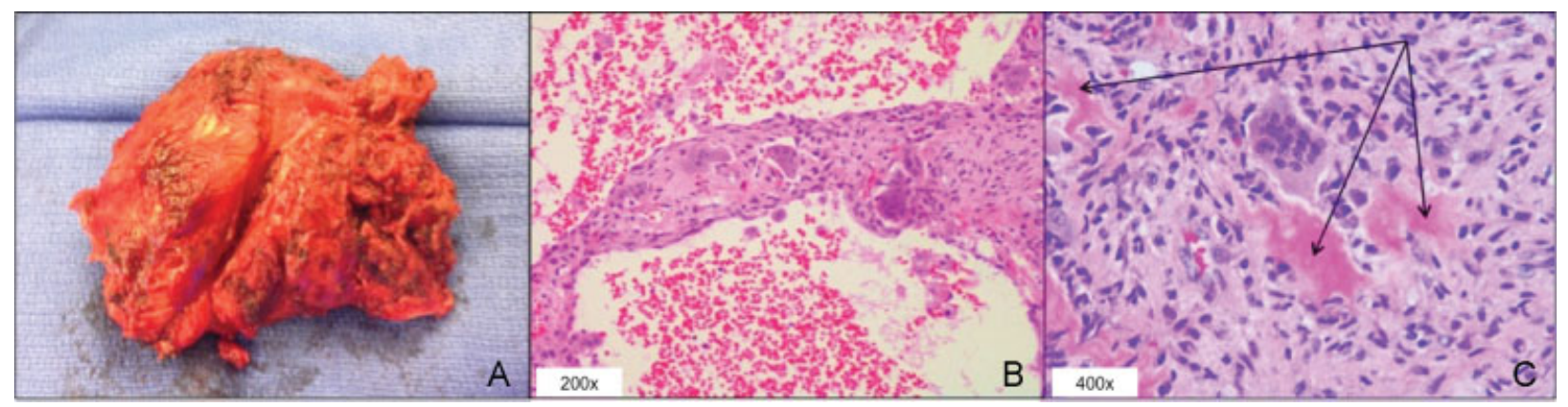

Fig. 2 Gross photograph of $A B C(A)$. H\&E (Hematoxylin and Eosin) staining showing septa consisting of spindle cells and multinucleate giant cells, separating blood-filled spaces(B) and solid area of $A B C$, with osteoid (woven bone) formation (black arrows) (C). 
take place and cause local inflammation further complicating resection. We performed an en bloc resection as described above with chest wall reconstruction via plate fixation and clavicle reimplantation, while preserving adjacent vessels and brachial plexus. In the end, this resulted in a short hospital stay of 2 days and full resolution of symptoms.

This case report illustrates a very rare cause of thoracic outlet syndrome arising from an even more uncommon location.

\section{Funding}

This work was not funded.

\section{Conflicts of Interest}

None declared.

\section{Acknowledgment}

None.

\section{References}

1 Brantigan CO, Roos DB. Etiology of neurogenic thoracic outlet syndrome. Hand Clin 2004;20(1):17-22

2 Leithner A, Windhager R, Lang S, Haas OA, Kainberger F, Kotz R. Aneurysmal bone cyst. A population based epidemiologic study and literature review. Clin Orthop Relat Res 1999;(363):176-179

3 Cottalorda J, Kohler R, Sales de Gauzy J, et al. Epidemiology of aneurysmal bone cyst in children: a multicenter study and literature review. J Pediatr Orthop B 2004;13(6):389-394

4 Hughes EK, James SL, Butt S, Davies AM, Saifuddin A. Benign primary tumours of the ribs. Clin Radiol 2006;61(4):314-322

5 Cottalorda J, Bourelle S. Current treatments of primary aneurysmal bone cysts. J Pediatr Orthop B 2006;15(3):155-167 